\title{
Vitesses et modalités de recul des falaises crayeuses de Haute-Normandie (France) : méthodologie et variabilité du recul
}

\section{Retreat rates and retreat modalities of coastal chalk cliffs in Upper Normandy (France): methods and retreat variability}

\author{
Pauline Letortu*, Stéphane Costa*, Abdelkrim Bensaid*, Jean-Michel Cador*, Hervé Quénol**
}

\section{Résumé}

Les falaises de Haute-Normandie connaissent une intense dynamique régressive qui a surtout été étudiée à travers le suivi du haut de falaise. La comparaison de l'orthophotographie de 2008 (BD Ortho ${ }^{\circledR}$, IGN) avec l'analyse photogrammétrique initiale (1966-1995 ; Costa, 2000) fournit des résultats sur une période plus longue (1966-2008, soit 42 ans). Ces vitesses ont été calculées tous les $50 \mathrm{~m}$ pour mettre en évidence des phénomènes de grande échelle, notamment l'influence des obstacles comme les éboulements/écroulements majeurs ou les jetées portuaires, induisant une multiplication par trois à quatre de l'ablation. Au-delà de l'approche classique de l'estimation des vitesses de retrait, une évaluation des modalités de recul des falaises crayeuses (fréquence et intensité) a été menée entre Étretat et Le Tréport grâce au suivi des éboulements/écroulements recensés sur les photographies aériennes verticales de l'IGN préalablement géoréférencées (1939-1995) et sur les orthophotographies (2000 et 2008). Un inventaire des éboulements/écroulements entre 2002 et 2009 (association ESTRAN) et sur 37,5 km de côte complète les informations obtenues. Associées aux vitesses d'ablation, ces valeurs permettent de préciser les modalités d'évolution de ces côtes à falaises. Schématiquement, deux secteurs aux dynamiques distinctes sont observés. Le premier secteur (cap d'Antifer/Saint-Valéry-en-Caux et Dieppe/Le Tréport) a des vitesses de recul faibles à modérées (de 0,09 à $0,18 \mathrm{~m} / \mathrm{an}$ ) et se caractérise par des éboulements/écroulements rares mais volumineux (médiane de l'aire de départ de l'éboulement/écroulement de $700 \mathrm{~m}^{2}$ pour Fécamp/Életot). Le second secteur (Saint-Valéry-en-Caux/Dieppe) est affecté par des vitesses de recul plus importantes $(0,23 \mathrm{~m} / \mathrm{an})$, et les éboulements/écroulements y sont plus fréquents mais peu volumineux (médiane de l'aire de départ de l'éboulement/écroulement de $200 \mathrm{~m}^{2}$ pour Quiberville/Dieppe). Grâce au Service Littoral de l'association ESTRAN, l'inventaire hebdomadaire des éboulements/écroulements réalisé entre Veules-les-Roses et Le Tréport (2002-2009) fournit statistiquement des éléments de réponse quant à la fréquence d'occurrence et les périodes de retour des éboulements/écroulements (ajustements à la loi de puissance). Ces valeurs corroborent les mesures de recul établies par photo-interprétation puisque là encore, le secteur du cap d'Ailly apparaît comme le plus dynamique : un éboulement/écroulement de plus de $1000 \mathrm{~m}^{3}$ par km linéaire est attendu tous les 6 mois contre tous les 2 à 3 ans pour les autres secteurs (Veules-les-Roses/Le Tréport, Hautot-sur-Mer/Bracquemont). Les informations obtenues présentent un grand intérêt pour la cartographie de l'érosion côtière.

Mots clés : falaises, érosion côtière, Haute-Normandie, géomorphologie littorale, éboulement/écroulement, photo-interprétation, analyse spatiale.

\begin{abstract}
The cliffs of Upper Normandy have high erosion dynamics which have mostly been studied by monitoring the cliff top. By integrating the orthophotograph of 2008 (BD Ortho ${ }^{\circledR}$, IGN) to the initial photogrammetric analysis (1966-1995, Costa, 2000), this work provides results over a longer time period (1966-2008, so 42 years). To determine the rate of cliff top retreat, two calculation methods on fine scale (every $50 \mathrm{~m}$ ) were tested: the "baseline approach" and the "area-based approach". These retreat rates were calculated every 50 $m$ in order to highlight the fine scale phenomena (especially the influence of shingle transit obstacles as major falls or port piers leading to multiplication by 3 to 4 of ablation rates). Beyond the traditional approach of estimating retreat rates, a work about retreat modalities was conducted between Etretat and Le Treport thanks to the inventory of falls on vertical aerial photographs of National Geographic Institute (IGN) (1939-1995), previously georeferenced, and orthophotographs (2000 and 2008). An inventory of falls from 2002 to 2009 (ESTRAN non-profit organization) along $37.5 \mathrm{~km}$ of coast provides additional information. Associated to rates, these val-
\end{abstract}

\footnotetext{
* LETG-Caen Géophen - Université de Caen Basse-Normandie - UMR LETG 6554 CNRS - Esplanade de la paix - BP 5186 - 14032 Caen Cedex 5 France (pauline.letortu@unicaen.fr ; stephane.costa@unicaen.fr ; abdelkrim.bensaid@unicaen.fr ; jean-michel.cador@unicaen.fr).

** LETG-Rennes Costel - Université de Rennes 2 Haute-Bretagne - UMR LETG 6554 CNRS - Campus Villejean, Maison de la Recherche - 6 avenue Gaston Berger - 35043 Rennes Cedex - France (herve.quenol@uhb.fr).
} 
ues bring information about evolution modalities, two areas with different dynamics are observed. The former area (Antifer cape/Paluel and Dieppe/Le Treport) has low to moderate retreat rates (from 0.09 to $0.18 \mathrm{~m} / \mathrm{y}$ ) and is characterized by rare but large falls (median of departure area of falls of $700 \mathrm{~m}^{2}$ for Fecamp/Eletot). The latter sector (Saint-Valery-en-Caux/Dieppe) has greater retreat rate $(0.23 \mathrm{~m} / \mathrm{y})$ and falls are more frequent but not massive (median of departure area of falls of $200 \mathrm{~m}^{2}$ for Quiberville/Dieppe). However, thanks to the Service Littoral (ESTRAN non-profit organization), weekly fall inventory from Veules-les-Roses to Le Treport (2002-2009) statistically provides some answers about the occurrence frequency of falls and their return periods (fitting to the power law). This information corroborates retreat results obtained by photo-interpretation because, again, the area of Ailly cape appears as the most dynamic with a fall over 1,000 $\mathrm{m}^{3}$ per linear $\mathrm{km}$ expected every 6 month whereas it is from 2 to 3 years for other sectors (Veules-lesRoses/Le Treport, Hautot/Bracquemont), with a retreat rhythm (elapsed time between two successive falls at the same place) estimated at 7 years. The obtained information is of great interest for mapping coastal erosion.

Key words: cliff, coastal erosion, Upper Normandy, coastal geomorphology, fall, photo-interpretation, spatial analysis.

\section{English abridged version}

The studied cliff coasts, located in the north-western termination of the Paris Basin, extend $100 \mathrm{~km}$ from Antifer cape (southwest) to Le Treport (northeast). Cliffs (average 60 to $70 \mathrm{~m}$ high) are made of upper Cretaceous chalk (from Cenomanian to Campanian), more or less resistant to weathering (Megnien and Megnien, 1980; Pomerol et al., 1987). Residual formations with flint and loess of Quaternary age (Lautridou, 1985) are located on this highly karstic area (Rodet, 1991). These cliffs are associated with two other coastal landforms: 1) a shore platform from 150 to $350 \mathrm{~m}$ wide hidden at the top by 2) a thin shingle barrier (30 $\mathrm{m}$ to $100 \mathrm{~m}$ wide).

This paper aims to contribute to the quantification of rates and modalities (frequency/intensity) in chalk cliff retreat. The focuses are about, firstly the choice of a method for the retreat quantification (fine scale: every $50 \mathrm{~m}$ and on multidecadal time), and secondly, about the modalities of cliff retreat from geomorphological markers observable on IGN vertical aerial photographs and on the field (inventory of ESTRAN non-profit corporation). The modalities are rarely studied, whereas they are essential in terms of risk management, and about the understanding of evolutionary trends (potential speeding up retreat rates).

The retreat rates of coastal chalk cliffs of the Seine-Maritime (Upper Normandy) have always attracted interest (De Lamblardie, 1789) and are important for risk management (risk areas planning). The choice of the coastline reference is essential and it is the top of the chalk cliff for this study. Various sources to monitor the cliff top are used (cadastral registers, vertical aerial photographs, photogrammetric surveys, airborne laser surveys...). Quantification of retreat rates every $50 \mathrm{~m}$ must be based on data of high reliability but also over many decades. On the area Antifer cape/Le Treport, photogrammetric data (1966-1995) and those from the orthophotograph of 2008 appear to be the most suitable ones $(0.50 \mathrm{~m}$ resolution) for calculating retreat rates over 42 years (19662008). The choice of the method of retreat rate calculating is essential and must be adapted to the specificities of places, such as the particularly irregular coastline between Antifer cape and Fecamp. Generally, the baseline approach proposed by R. Dolan et al. (1978) and generalized by E.R. Thieler et al. (2005) with DSAS extension for ArcGIS ${ }^{\circledR}$, is a method of point monitoring advocated by many authors (Faye et al., 2012; Foyle and Naber, 2012). The latter method, based on lost area between successive coastlines, has the advantage to follow the retreat in a spatially continuous way (Costa, 2000).

Due to the complexity of the design of Upper Normandy cliffs, the baseline method does not seem appropriate. Indeed, nonparallel evolution of the cliff edge between two known states leads to many non-perpendicular transect measurements to the "coastline". These frequent cases overestimate retreat rate. The calculation of the lost area is more relevant (area-based approach). The average rate of retreat between Antifer cape and Le Treport is estimated at $0.15 \mathrm{~m} / \mathrm{y}( \pm 0.03 \mathrm{~m} / \mathrm{y})$ over the period 1966-2008. The estimated retreat every $50 \mathrm{~m}$ shows that this average masks a high spatial variability of cliff retreat.

On the scale of hydrosedimentary cells, notably delimited by port piers, it is possible to recognize two areas of contrasted evolution rates: (1) from Antifer cape to Fecamp, where rates are relatively low $(0.09$ to $0.10 \mathrm{~m} / \mathrm{y})$, and a large area of moderate retreat (from 0.12 to $0.18 \mathrm{~m} / \mathrm{y}$ ) from Fecamp to Saint-Valery-en-Caux and from Dieppe to Le Treport ; (2) a sector with high retreat $(0.23 \mathrm{~m} / \mathrm{y})$, between Saint-Valery-en-Caux and Dieppe. These results confirm those of S. Costa $(1997,2000)$ and S. Costa et al. (2004) which suggested a close relationship with the regional lithology. Areas where Santonian and Campanian chalk outcrops (Saint-Valery-en-Caux/Dieppe), retreat rates are higher than where the Cenomanian, Turonian and Coniacian chalk outcrops (cap d'Antifer/Saint-Valery-en-Caux or Dieppe/Le Treport), marked by moderate rates.

On fine scale (retreat data every $50 \mathrm{~m}$ ), retreat rates of chalk cliff top have an even greater spatial variability. The accuracy of data also shows at the immediate downdrift side of major falls and port piers (along few hundred meters) average rates can be multiplied by 3 to 4 because of sediment deficit induced by shingle transit obstacles.

With the rates of retreat, the retreat modalities of the cliffs are essential for a better understanding of cliff erosion.

To account for these modalities, visible falls on IGN aerial photographs, that were previously georeferenced, were digitized at 1/2,000 and analyzed. The inventory of geomorphological markers (whitish scars visible on the cliff top) has the advantage to provide some answer elements about (1) possible changes in time and space, intensity or the frequency of these events (via the determination of the number of falls by aerial surveys), (2) the median or maximum retreat per fall per sector. 
In total, 599 falls (deposit area) were recorded between 1939 and 2008. The spatial distribution of these events is not homogeneous: a high density (up to 19 falls per linear $\mathrm{km}$ between 1939 and 2008) is observed between Sennevillesur-Fecamp and Veulettes-sur-Mer, between Ailly cape and Dieppe, between Dieppe and Belleville-sur-Mer and at the north of Penly. With the exception of Saint-Valery-en-Caux, where the number of aerial surveys available is very low, it is proved that the frequency of events is particularly important at the downdrift side of obstacles to shingle transit.

The cartography of the fall departure and deposit places allows us to measure their area. For example, the departure areas are particularly important in coastal sections Fecamp / Eletot (the area of the fall can reach 6,000 $\mathrm{m}^{2}$ ) and Dieppe / Criel-sur-Mer (maximum between 1,800 and 5,800 $\mathrm{m}^{2}$ ). Between Saint-Valery-en-Caux and Dieppe, the areas are small (maximum of 1,500 $\mathrm{m}^{2}$ ).

An increase in the number of falls since 2000 is noticeable, but it is difficult to identify any tendency because increased resolution of photographs allows the observation of more falls than before.

However, the quantification of rates and modalities by area-based method and by the inventory of falls (frequency and area) highlights two sectors with different dynamics: 1) an area with low and moderate retreat rates (from 0.09 to $0.18 \mathrm{~m} / \mathrm{y}$ ) characterized by rare but large falls (Antifer / Paluel and Dieppe / Le Treport); 2) a sector (Saint-Valeryen-Caux / Dieppe) with greater retreat rate $(0.23 \mathrm{~m} / \mathrm{y})$ characterized by more frequent falls but not massive. This spatial distribution of dynamics is to relate with the morphostructural characteristics of Upper Normandy (Costa, 1997; Costa et al., 2004). Thus, the coastal section SaintValery-en-Caux/Dieppe, located in the axis of the "Norman trough", is characterized by low cliffs carved into Santonian and Campanian chalk with low resistance to weathering (Laignel, 1997).

Regarding the estimation of the return period of falls, it was necessary to resort to another more accurate method but on a smaller coastline. It is a weekly survey of all observable coastal cliff falls (from several $\mathrm{m}^{3}$ to tens or even hundreds of thousands of $\mathrm{m}^{3}$ ) between Veules-les-Roses and Le Treport (about $37.5 \mathrm{~km}$ of coast). This inventory was conducted by ESTRAN non-profit corporation of Dieppe, between 2002 and 2009.

The 331 falls recorded between 2002 and 2009 allow us to make an adjustment to the power law commonly used for this type of phenomenon (Lim et al., 2010; Dewez et al., 2013). This statistical approach is to determine the return period of falls in function of the fallen volume. A fall over $1,000 \mathrm{~m}^{3}$ per linear kilometer is expected every 6 month between Sainte-Marguerite-sur-Mer to Varengeville-sur-Mer, whereas it is 2.5 year for the sector Hautot-sur-Mer / Bracquemont. So the return period of falls is much faster than elsewhere in Ailly cape, except at the immediate downdrift side of obstacles to shingle transit. The fast retreat area in Ailly cape is also characterized by frequent falls but not massive because of fast retreat rhythm of the cliff estimated at 7 years. Among the factors responsible for these retreats, local morphostructural characteristics seem predominant: outcrop of Santonian and Campanian chalk considered most susceptible to weathering, low height of the chalk cliffs, leading to fast evacuation of falls and thus reactivating cliff instability, and the existence of perched water tables in the Eocene sandy and clayey formations which saturating chalk in water (at least superficially).

\section{Introduction}

L'intérêt porté à la dynamique régressive des falaises crayeuses haut-normandes n'est pas un fait nouveau (De Lamblardie, 1789) mais la quantification précise des vitesses et des rythmes de recul reste d'actualité, notamment pour la gestion du risque d'érosion côtière. L'étude de la dynamique régressive peut se faire à l'aide de diverses sources de données, dont la comparaison permet d'obtenir des vitesses. Parmi les documents couramment utilisés pour les falaises crayeuses, nous retrouvons les cadastres (Bialek, 1969 ; Hénaff et al., 2002 ; Dornbusch et al., 2008a ; Letortu, 2013), les photographies aériennes verticales (May et Heeps, 1985 ; Costa 1997 ; Hénaff et al., 2002 ; Lahousse et Pierre, 2003a et b ; Dornbusch et al., 2008a), les levés photogrammétriques (Lahousse et Pierre, 2003b ; Costa et al., 2004 ; Dornbusch et al., 2008b ; Letortu, 2013), les levés laser terrestre ou aérien (Dewez et al., 2013), ou encore les données issues de la datation au ${ }^{10} \mathrm{Be}$ de la plateforme d'érosion marine (Regard et al., 2012). Cependant, les marges d'erreur inhérentes à la précision des documents utilisés, aux méthodes et techniques choisies pour quantifier les évolutions, ou encore aux divers pas de temps d'analyse, font que les résultats sont parfois difficilement comparables, voire discutables.

Cet article a pour but de contribuer à la quantification des vitesses et des modalités de recul des falaises crayeuses. L'objectif de ce travail est double. Dans un premier temps, il s'agit de présenter la méthode préconisée pour la quantification de l'ablation à partir des données photogrammétriques de 1966 et 1995 (Costa, 2000), et de l'orthophotographie 2008 (BD Ortho ${ }^{\circledR}$ IGN). Dans un second temps, l'étude des modalités du recul des falaises (fréquence, intensité et répartition spatiale) s'appuie sur des marqueurs géomorphologiques observables 1) sur les photographies aériennes verticales et 2) sur le recensement hebdomadaire (2002-2009) de tous les éboulements/écroulements sur près de $37,5 \mathrm{~km}$ de côte (réalisé en collaboration avec le Service Littoral de l'association ESTRAN). Ce travail propose des éléments de réponse quant au rapport fréquence/intensité des phénomènes observés, mais également sur d'éventuelles augmentations des vitesses d'évolution.

\section{Présentation du secteur d'étude}

Les falaises haut-normandes, d'une altitude moyenne de 60 à $70 \mathrm{~m}$, s'étendent sur $100 \mathrm{~km}$ du cap d'Antifer au sudouest, jusqu'au Tréport au nord-est (Seine-Maritime). À leur pied se développe une plateforme d'érosion marine (de 150 à $350 \mathrm{~m}$ de largeur), masquée, en haut de plage, par un mince cordon de galets de silex. Les abrupts crayeux sont entre- 
coupés par de nombreuses vallées drainées ou sèches perpendiculaires au rivage, protégées à leur débouché par un cordon de galets souvent large (30 m à 100 m de largeur) en raison de la présence d'épis ou de jetées portuaires.

Situées à la terminaison nord-occidentale du Bassin parisien sédimentaire et au contact avec la Manche, les falaises cauchoises sont constituées de craies, datées du Crétacé supérieur (Mégnien et Mégnien, 1980 ; Pomerol et al., 1987). Sur ce bâti fortement karstifié (Rodet, 1991) reposent des formations résiduelles à silex et des lœss du Quaternaire (Lautridou, 1985). Les principales déformations tectoniques, de direction NO-SE, ont porté à l'affleurement divers étages géologiques du Crétacé supérieur (fig. 1). Les différents étages de craies possèdent de légères variations de faciès et des discontinuités sédimentaires de détail qui engendrent quelques subtils contrastes de résistance (Juignet et Breton, 1992 ; Laignel, 1997). Du plus ancien au plus récent, nous retrouvons les craies du Cénomanien, qui affleurent sur quelques mètres à la base des falaises du cap d'Antifer et d'Étretat, ainsi qu'à l'est de Fécamp. Ces dernières sont hétérogènes, parfois riches en éléments détritiques (argiles et quartz) et peuvent être glauco- nieuses ou noduleuses. Le Turonien est l'étage sus-jacent. Il est constitué de craies argileuses, grisâtres à blanchâtres, avec des bancs noduleux fréquents, et peu ou pas de silex. Cet étage affleure du cap d'Antifer à Étretat, de Fécamp à Életot, et de Puys (est de Dieppe) au Tréport. Il atteint son extension maximale à Penly au droit de l'anticlinal de Berneval-Londinières. Le Turonien est surmonté par la craie du Coniacien, qui affleure entre le cap d'Antifer et Saint-Valéry-en-Caux, ainsi qu'entre Dieppe et Le Tréport. L'étage sus-jacent du Santonien, apparaît dans la partie centrale du secteur étudié entre Saint-Valéry-en-Caux et Puys. Enfin, la craie du Campanien, la plus récente, est peu présente et ne se retrouve qu'entre Quiberville et Pourville-sur-Mer (Hautot-sur-Mer). Au regard d'études géotechniques, ces dernières (Santonien et Campanien), semblent plus favorables à la météorisation que les autres étages du Crétacé supérieur (Laignel, 1997). Au-dessus de ces assises crayeuses, une couverture de sédiments argileux et sableux d'environ 10 à $30 \mathrm{~m}$ d'épaisseur, d'âge paléogène (Bignot, 1962), se substitue à l'habituelle formation résiduelle à silex (Laignel, 1997 ; Costa et al., 2006), notamment au cap d'Ailly et à Sotteville-sur-Mer.

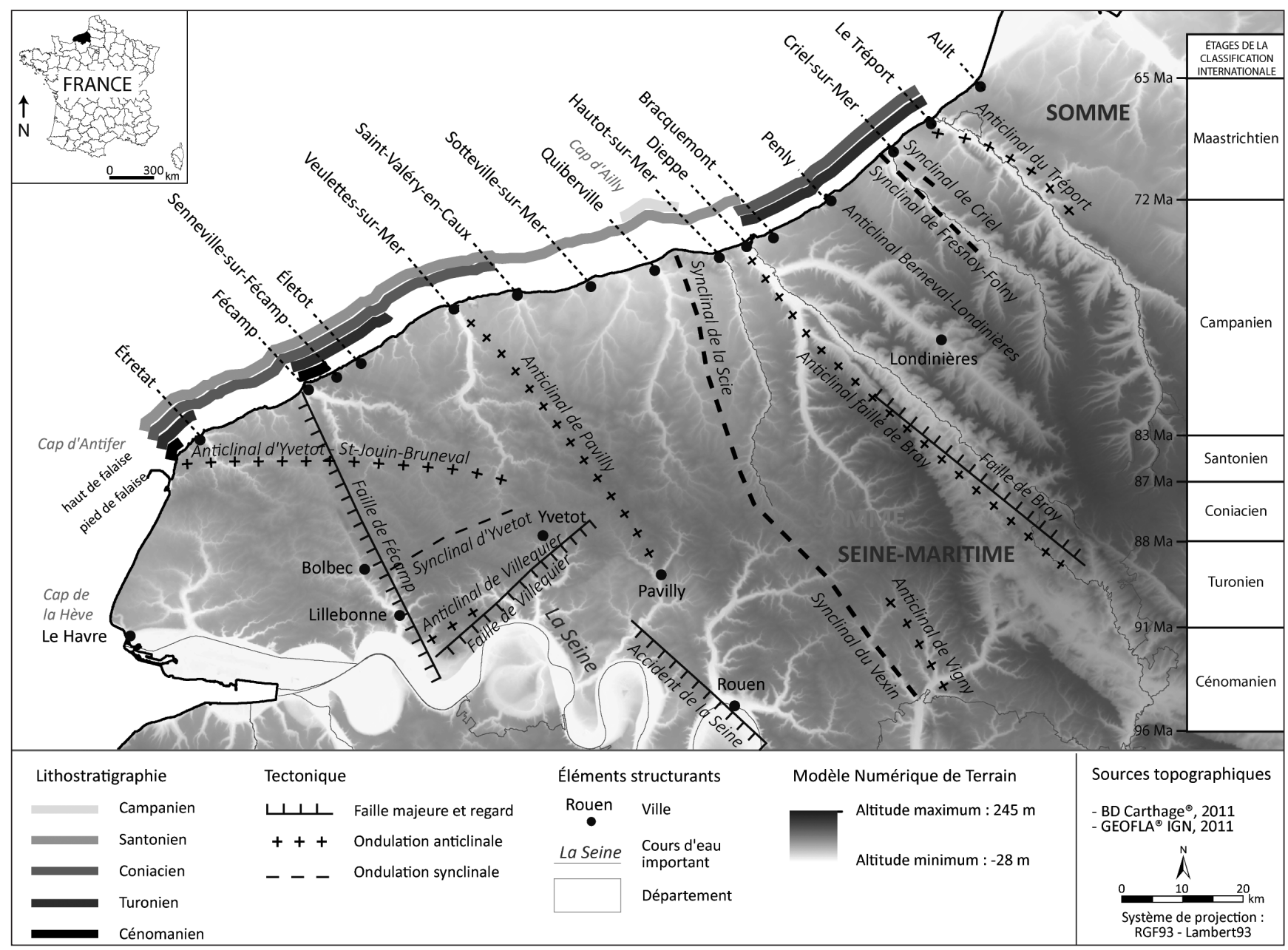

Fig. 1 - Schéma structural et lithostratigraphie simplifiéen en Haute-Normandie (d'après Mégnien et Mégnien, 1980 in Costa, 1997 ; Laignel, 1997).

Fig. 1 - Simplified structural map and lithostratigraphy characteristics in Upper Normandy (from Megnien et Megnien, 1980 in Costa, 1997; Laignel, 1997). 
La dynamique régressive des falaises de Haute-Normandie se traduit par des mouvements de masse instantanés, affectant tout ou partie de l'abrupt (éboulement/écroulement), ou des phénomènes d'éboulisation discontinue (essentiellement due à la cryoclastie). Le terme « écroulement » désigne le détachement d'un panneau de craie sans basculement, et «éboulement » lorsque la chute se prolonge par un déplacement roulant après l'impact (Hénaff et al., 2002). Au pied de ces abrupts se développent de fins cordons de galets (quelques mètres d'épaisseur tout au plus). Le cheminement et la répartition de ces derniers sont perturbés par les mouvements de masse et les jetées, qui constituent des obstacles au transit des galets. Leur absence ou leur faiblesse en pied de falaise sont susceptibles de modifier l'efficacité des actions marines, et par conséquent, les vitesses et les modalités du recul.

\section{Approche méthodologique}

\section{Le choix de l'indicateur morphologique de référence}

Au préalable de toute étude diachronique d'un rivage, la question du choix de la ligne de référence se pose. Elle doit être lisible sur tous les documents utilisés. Pour les côtes à falaises, l'indicateur morphologique de référence est, selon les sites et les auteurs, le pied ou le haut de falaise (May et Heeps, 1985 ; Costa, 1997, 2000 ; Lim et al., 2005 ; Pierre et Lahousse, 2006 ; Young et al., 2009). Le haut de falaise est fréquemment retenu car il est plus facilement observable sur les clichés verticaux. Pour ce travail, le haut de « l'abrupt crayeux » sera donc notre référentiel.

\section{La nécessité de documents précis et géoréférencés}

L'utilisation des cadastres napoléoniens (d'échelle $1 / 1000$ à 1/2 500), dont les plus anciens remontent aux années 1820 , permettraient d'obtenir un intervalle de temps important et donc plus représentatif. Cependant, les marges d'erreur de positionnement du haut de la falaise sont inconnues. Les photographies aériennes verticales de l'IGN, quant à elles, constituent une source de données plus fiable mais en l'absence de toute rectification, les perturbations géométriques sont nombreuses induisant des marges d'erreur plurimétriques (Moore, 2000 ; Robin, 2002). Deux procédures permettent de réduire ces marges d'erreur : l'orthorectification et le géoréférencement. Testées sur tout ou partie des missions aériennes de l'IGN disponibles entre 1939 et 1995, l'imprécision reste forte (au maximum de $\pm 6 \mathrm{~m}$ ) et la comparaison de longs linéaires côtiers de falaise, même à recul rapide, reste illusoire (Costa et al., 2004 ; Letortu, 2013).

L'établissement d'un levé photogrammétrique par voie numérique a connu une utilisation croissante au début des années 2000 et constitue des documents fiables (Overton et al., 1996 ; Costa, 2000 ; Moore, 2000 ; Lahousse et Pierre, 2003a et b ; Zviely et Klein, 2004). Pour le littoral haut-nor- mand, nous disposons de données photogrammétriques réalisées à partir des missions IGN qui le permettaient, à savoir 1966 et 1995 (Costa, 2000). Cette méthode fournit des résultats d'une grande précision pour la cartographie du « trait de côte » $( \pm 0,40 \mathrm{~m})$, mais également, des données numériques, géoréférencées et homogènes. Dans ce présent article, la représentativité temporelle de l'analyse du recul des falaises a été améliorée par l'intégration de l'orthophotographie de l'IGN (2008), dont la marge d'erreur du positionnement du haut de falaise est estimée à $\pm 1 \mathrm{~m}$ (Letortu, 2013). Au-delà d'une représentativité temporelle plus grande, cette nouvelle couche d'information permet de proposer une première approche diachronique (1966-1995-2008) et donc d'apporter des éléments de réponse quant à une éventuelle évolution des vitesses de recul.

\section{De l'intérêt de la quantification des surfaces perdues en haut de falaise}

Pour garantir une plus grande homogénéité, la digitalisation manuelle du haut de falaise a été réalisée sur le même ordinateur, par le même opérateur, et en suivant une grille de photo-interprétation lors de la digitalisation.

Deux méthodes principales sont communément proposées pour quantifier le recul des côtes. La première, appelée « approche de la ligne de base » ou « approche des transects » a été préconisée par de nombreux auteurs (Dolan et al., 1978 ; Faye et al., 2012 ; Foyle et Naber, 2012). Cette dernière est souvent utilisée en raison de l'existence d'un module de mesure automatique DSAS (Thieler et al., 2005), qui simplifie la quantification des vitesses. Il s'agit ici de mesurer la longueur de transects joignant les traits de côtes de dates différentes à partir d'une ligne de base sensiblement parallèle au référentiel le plus récent. La méthodologie adoptée peut se décomposer en trois étapes. La première a pour but de créer une "ligne de base ». Pour ce faire, le trait de côte de 2008 est dédoublé et situé $1 \mathrm{~m}$ en arrière vers les terres. La seconde étape consiste à générer des transects perpendiculaires à la ligne de base, espacés de $50 \mathrm{~m}$. La dernière étape vise à calculer la mobilité du trait de côte pour chaque transect, et pour chaque date. Or, il s'agit d'une mesure ponctuelle, notamment de la longueur d'un transect entre deux traits de côte qui n'évoluent, à grande échelle, que rarement parallèlement. Cette mesure ponctuelle est finalement peu représentative de l'évolution du haut de falaise, souvent affecté par de grands mouvements de masse. C'est la raison pour laquelle nous proposons une seconde méthode : «l'approche fondée sur l'aire, qui mesure, de manière continue, le recul en haut de falaise en quantifiant les aires perdues entre deux dates. Conceptuellement, les lignes de référence (traits de côte à deux dates) sont intersectées (ou sectionnées) par des segments de droite disposés tous les $50 \mathrm{~m}$ et perpendiculairement au trait de côte le plus récent (Costa, 2000 ; Robin, 2002). La valeur de recul est obtenue en divisant ces aires (ou polygones) par le linéaire étudié et l'intervalle de temps entre les deux positions du haut de falaise (fig. 2). 

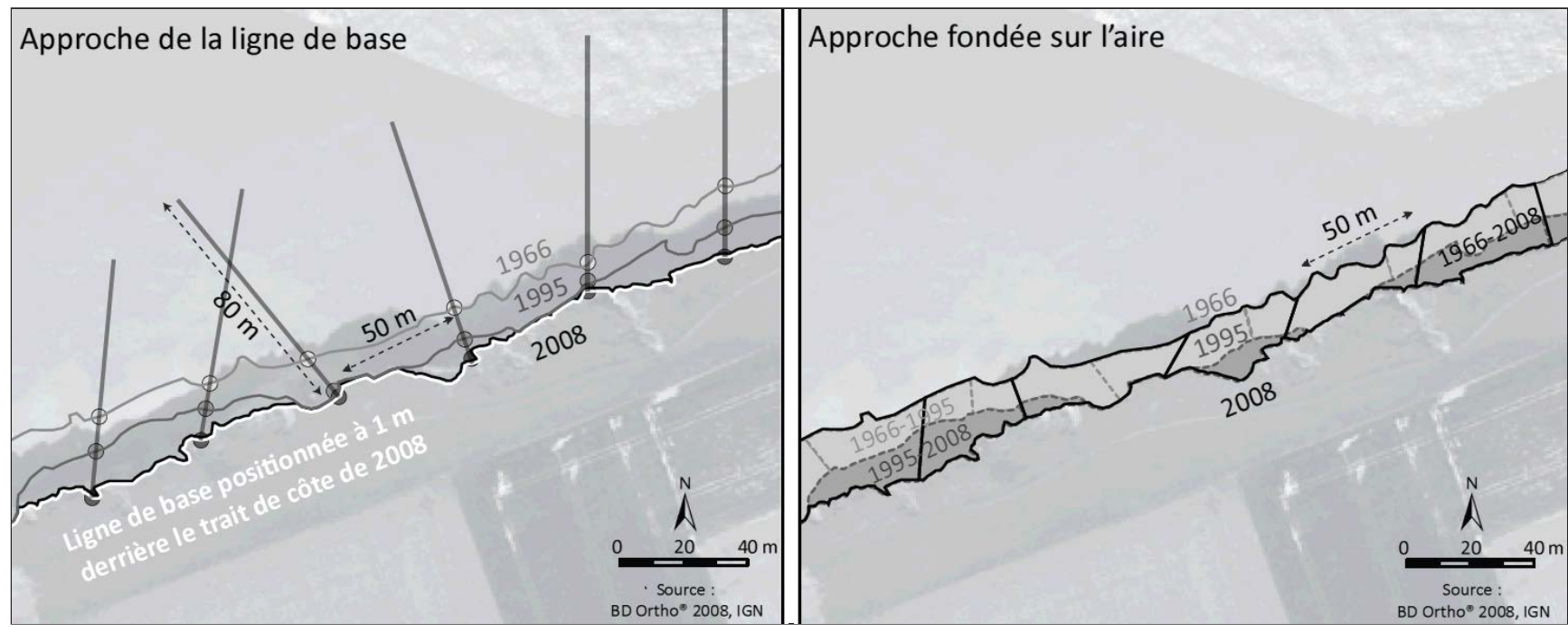

Fig. 2 - Méthodes de calcul du recul des falaises.

Fig. 2 - Methods of cliff retreat measurements.

\section{Première approche pour la définition des modalités (fréquence/intensité) voire des rythmes de recul des falaises}

Au-delà des vitesses de recul, il est également fondamental de comprendre la répartition spatiale de la fréquence et de l'intensité des mouvements gravitaires. Pour ce faire, les éboulements/écroulements visibles sur les photographies aériennes de l'IGN ont donc été comptabilisés. Cet inventaire présente l'avantage de fournir des informations sur (1) une éventuelle évolution, dans le temps et l'espace, de l'intensité et/ou de la fréquence de ces événements (via l'établissement du nombre d'éboulements/écroulements par mission aérienne), (2) le recul médian ou maximum par éboulement/écroulement et par secteur.

Ce travail de recensement est fondé sur la cartographie (au $1 / 2$ 000) des zones de départ des mouvements de masse observés sur les missions aériennes de l'IGN préalablement géoréférencées (de 1939 à 1995), et sur les orthophotographies de 2000 et 2008.

Par conséquent, 17 missions ont été utilisées entre 1939 et 2008 (soit 69 ans d'évolution). L'une des difficultés rencontrées réside dans l'absence de continuité spatiale dans les séries photographiques utilisées. Ainsi, des secteurs sont régulièrement couverts, d'autres comme Saint-Valéryen-Caux/Quiberville le sont très peu (uniquement par les orthophotographies de 2000 et 2008) (fig. 3). De plus, l'échelle d'observation peut faire manquer les petits éboulements / écroulements.

Face à cette difficulté, nous avons fait appel à une autre méthode plus précise spatialement mais sur un linéaire côtier et un laps de temps plus restreints (environ $37,5 \mathrm{~km}$ de 2002 à 2009). Il s'agit d'un levé hebdomadaire de tous les éboulements/écroulements (quel que soit leur volume) entre Veules-les-Roses et Le Tréport, réalisé par le Service Littoral de l'association ESTRAN. Il s'agit de relever précisément le lieu, la date et les mensurations de la zone de dépôt de l'éboulement/écroulement observé. Au total, 331 événements ont été recensés, allant de 1 à $236000 \mathrm{~m}^{3}$. Ceux de faible volume $\left(0-200 \mathrm{~m}^{3}\right)$ représentent $40 \%$ de la population totale mais seulement $1 \%$ du volume de débris produit (Letortu, 2013).

\section{Résultats}

\section{Vitesses de recul des falaises}

La quantification des aires perdues en haut de falaise entre deux états connus est plus pertinente. En effet, au regard des travaux existants, il s'avère que le calcul en un point (approche de la ligne de base ou des transects) aboutit à de fortes surestimations/sous-estimations de la mesure car les traits de côte évoluent rarement de façon perpendiculaire au transect généré.

La méthode fondée sur l'aire, du cap d'Antifer au Tréport, entre 1966 et 2008, est plus cohérente avec les observations de terrain. Ainsi, le recul moyen du haut de falaise « abrupt crayeux » est de l'ordre de $0,15 \mathrm{~m} / \mathrm{an}( \pm 0,03 \mathrm{~m} / \mathrm{an})$ contre $0,21 \mathrm{~m} / \mathrm{an}$ établi par S. Costa (2000). Cette valeur moyenne masque une grande variabilité spatiale, et ce, à diverses échelles.

À l'échelle des cellules hydro-sédimentaires, il est possible de reconnaître deux sections aux vitesses d'évolution contrastées (marge d'erreur $\pm 0,03 \mathrm{~m} / \mathrm{an}$ ) : (1) un secteur au recul faible $(0,09$ à $0,10 \mathrm{~m} / \mathrm{an})$ du cap d'Antifer à Fécamp et un modéré $(0,12$ à $0,18 \mathrm{~m} / \mathrm{an})$ de Fécamp à Saint-Valéry-enCaux, puis de Dieppe au Tréport ; (2) un secteur au recul fort (0,23 m/an) entre Saint-Valéry-en-Caux et Dieppe. Cette répartition spatiale des vitesses de recul rappelle celle évoquée par S. Costa (1997) et S. Costa et al. (2004), avec comme explication principale, une relation avec la lithologie régionale. Ainsi, dans les secteurs où affleurent les craies du Santonien et du Campanien (Saint-Valéry-en-Caux/Dieppe), les vitesses de recul sont les plus fortes, alors que là où affleurent le Turonien et la base du Coniacien (Étretat/SaintValéry-en-Caux et Dieppe/Le Tréport), l'ablation est plus 




Fig. 3 - Séries de photographies aériennes verticales disponibles (IGN) sur le secteur d'étude.

Fig. 3 - Available aerial photographs (IGN) for the study area.

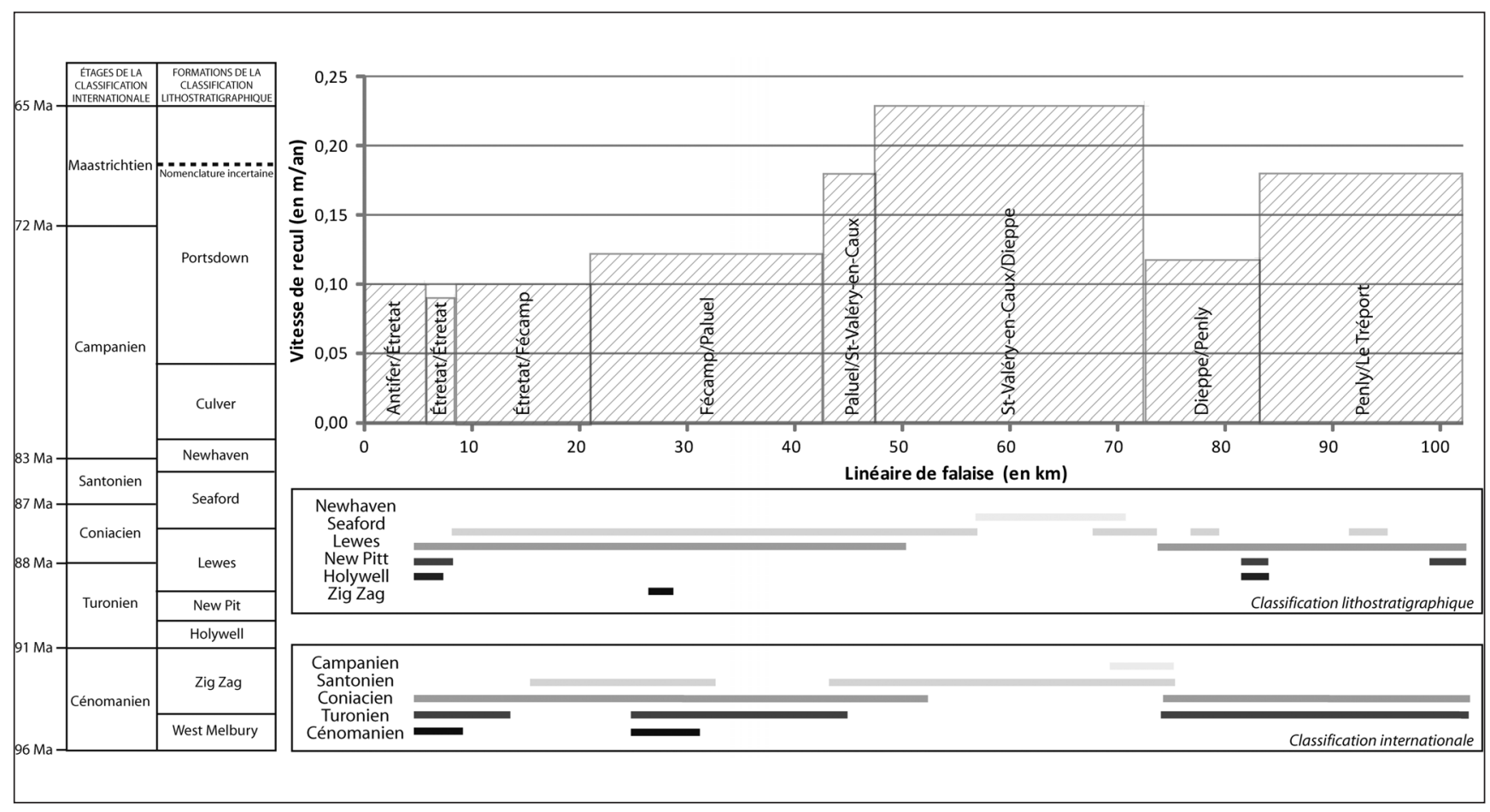

Fig. 4 - Vitesses de recul entre le cap d'Antifer et Le Tréport (1966-2008) et relation avec la lithologie.

Fig. 4 - Retreat rates from Antifer cape to Le Treport (1966-2008) linked to lithology characteristics. 


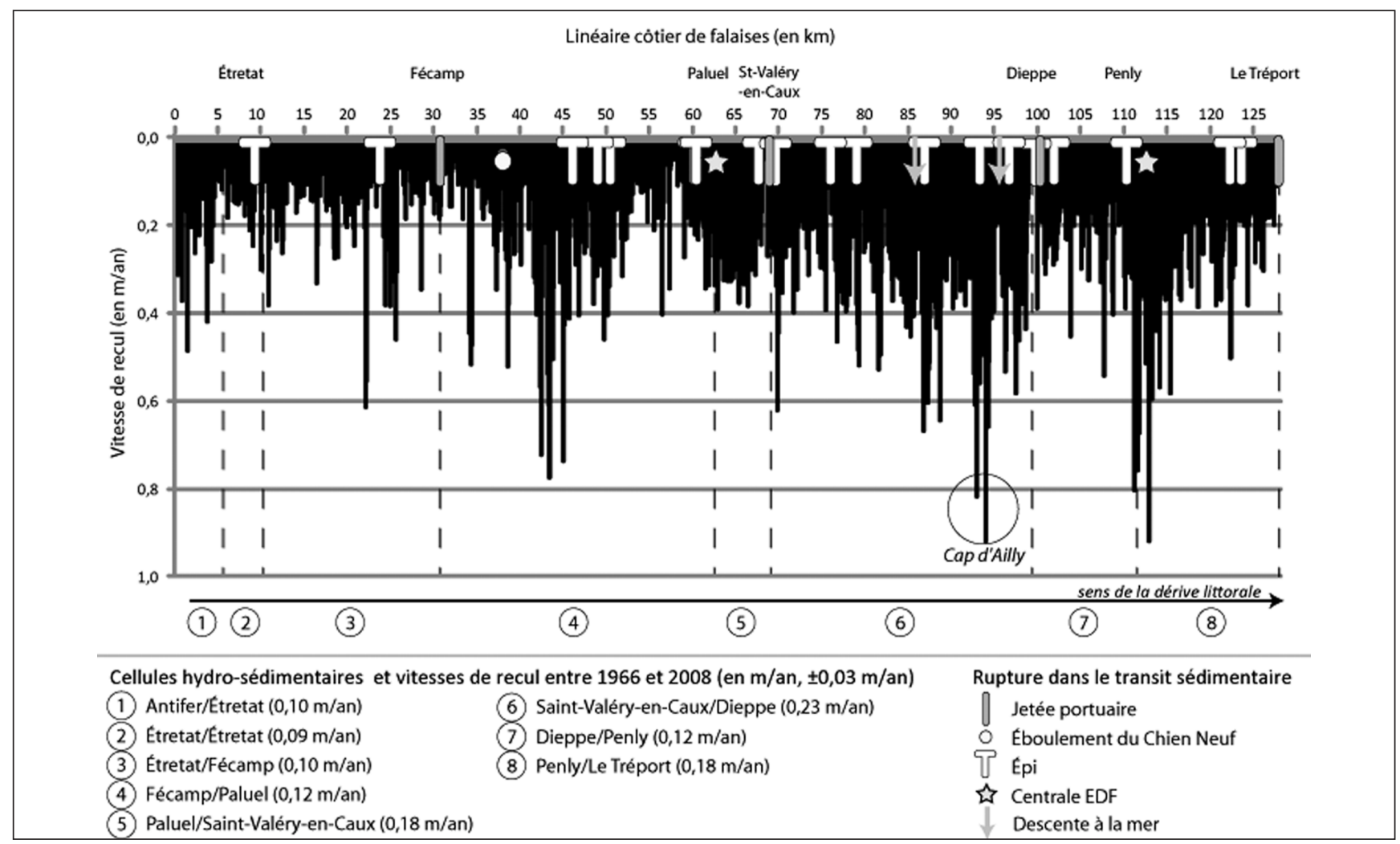

Fig. 5 - Impacts des obstacles sur les vitesses de recul des falaises haut-normandes (1966-2008), établies tous les $50 \mathrm{~m}$. Fig. 5 - Impacts of obstacles on retreat rates for coastal chalk cliffs in Upper Normandy (1966-2008), calculated every 50 m.

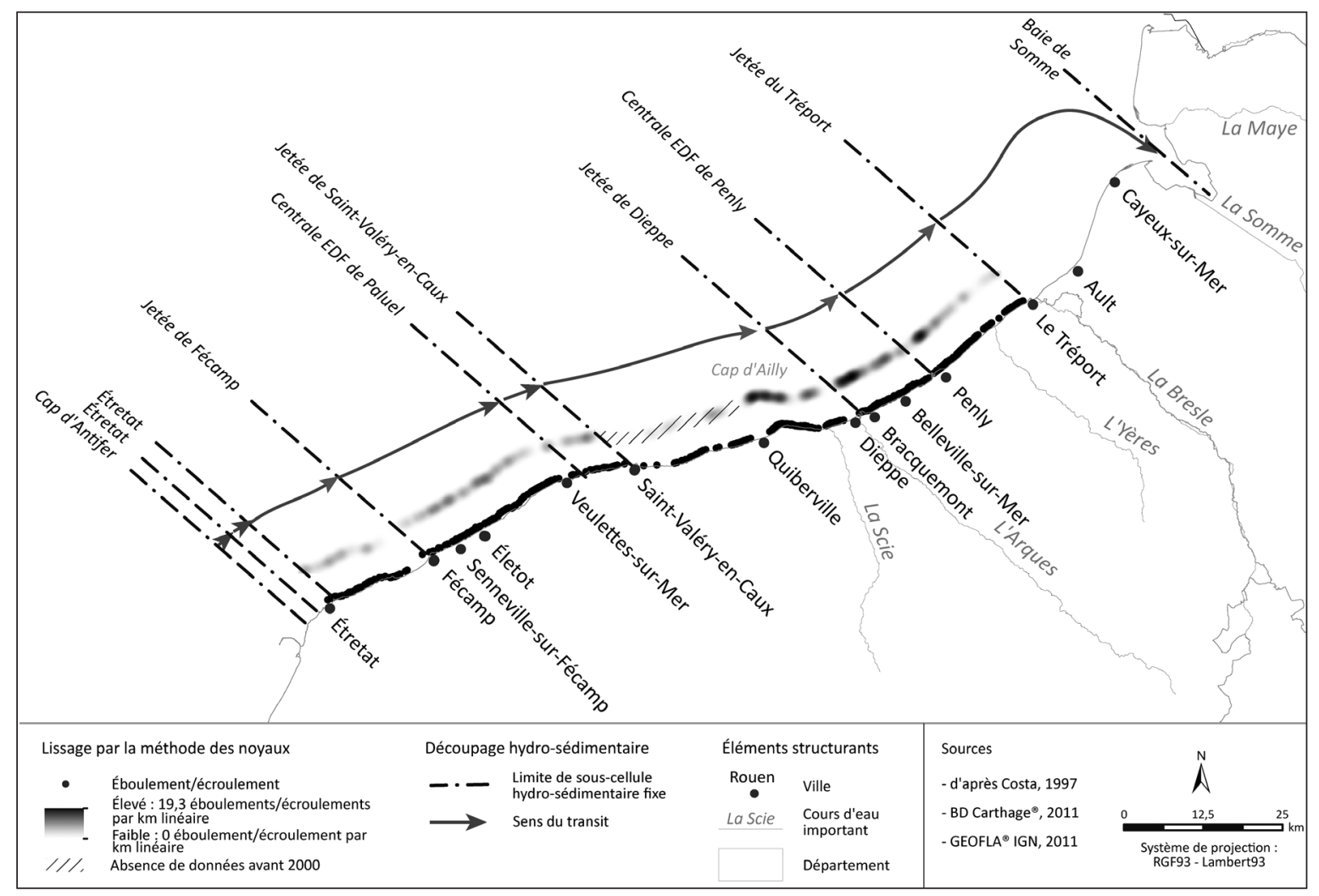

Fig. 6 - Densité (lissage par la méthode des noyaux) du nombre d'éboulements/écroulements recensés (par km linéaire) entre Étretat et Le Tréport (1939-2008).

Fig. 6 - Density (kernel) of falls number censed (per linear km) from Etretat to Le Treport (1939-2008). 
modérée. Il en est de même pour le secteur Antifer/Étretat, dont les reculs très faibles sont également probablement liés à l'existence de hard-grounds massifs, de nombreux lits de silex dans les craies et à l'affleurement du Cénomanien à la base des falaises (fig. 4).

Afin d'affiner cette lithodépendance, nous avons tenté de corréler la répartition spatiale des vitesses de retrait avec le découpage lithostratigraphique événementiel, établi par B. Pomerol et al. (1987). Cette approche n'apporte aucune nouvelle information (fig. 4).

À grande échelle (tous les $50 \mathrm{~m}$ ), les vitesses de recul de la falaise présentent une variabilité spatiale encore plus importante avec une accentuation systématique de ces dernières en aval-dérive immédiat des obstacles au transit des galets (fig. 5). Sur environ $400 \mathrm{~m}$ après l'obstacle (jetées portuaires et de centrales EDF, épis majeurs, éboulements/écroulements majeurs tels que celui du «Chien Neuf» à Senneville-surFécamp), les vitesses de recul sont trois à quatre fois supérieures à celles mesurées avant l'obstacle. Ces phénomènes observés sur la période 1966-1995 (Costa et al., 2004), perdurent, voire s'accentuent sur la période plus récente, 19952008. De plus, cette augmentation du retrait est proportionnelle à la date d'apparition de l'obstacle et semble progresser dans le sens de la dérive littorale telle une onde. L'existence de ces obstacles, qui perturbent le transit des galets semble exacerber localement l'efficacité des actions marines au pied de falaise. Cet état de fait perturbe également la sectorisation des vitesses de recul à l'échelle de la Seine-Maritime, qui semble en relation avec les caractéristiques lithologiques régionales. Ainsi, même dans les craies du Turonien, qui sont moins favorables à la météorisation, l'absence de galets en aval-dérive des obstacles majeurs, induit un fort accroissement des vitesses d'ablation.

\section{Les modalités et les rythmes de recul des falaises}

Cinq cent quatre-vingt-dix-neuf éboulements/écroulements ont été observés sur les diverses missions de photographies aériennes de l'IGN, entre Étretat et Le Tréport (1939-2008). Ces événements recensés sont spatialement inégalement répartis. La figure 6 présente la densité des phénomènes recensés par km linéaire (lissage par la méthode des noyaux qui calcule le nombre d'éboulements/écroulements dans un cercle ; Letortu, 2013). Elle fait apparaître des espaces marqués par une forte densité (jusqu'à 19 éboulements/écroulements par km linéaire entre 1939 et 2008), entre SennevilleFécamp et Veulettes-sur-mer, entre le cap d'Ailly et Dieppe, entre Dieppe et Belleville-sur-Mer, puis au nord de Penly. À l'exception de Saint-Valéry-en-Caux, où le nombre de missions disponibles est très faible, la fréquence des éboulements/écroulements est une fois de plus particulièrement importante en aval-dérive d'obstacles au transit des galets (fig. 6). Ainsi, nous observons une augmentation locale des vitesses, 3 à 4 fois supérieures à celles observées en amontdérive. Cela corrobore les résultats obtenus entre 1966, 1995 et 2008, par la méthode fondée sur l'aire.

Ce recensement des 599 éboulements/écroulements permet de s'interroger sur l'existence d'une répartition spatiale de l'ampleur des départs de matériaux en haut de falaise, mais également sur une possible évolution dans le temps de la fréquence de ces mouvements de masse.

Ce travail met en évidence que ces événements sont particulièrement importants sur les sections côtières de Fécamp / Életot (l'aire médiane des éboulements/écroulements en haut de falaise est de $675 \mathrm{~m}^{2}$ et le maximum peut atteindre $5800 \mathrm{~m}^{2}$ ), de Dieppe/Criel-sur-Mer (l'aire médiane est de l'ordre de $250 \mathrm{~m}^{2}$ et le maximum peut atteindre $1800 \mathrm{~m}^{2}$ ). En revanche, entre Quiberville/ Dieppe, les surfaces de départ sont encore plus faibles (médianes de $200 \mathrm{~m}^{2}$ et le maximum atteignant $1500 \mathrm{~m}^{2}$; fig. 7).

S'agissant du nombre d'éboulements/écroulements, aucune tendance significative n'apparaît entre 1947 et 2000. En revanche, depuis 2000, cette fréquence est beaucoup plus importante, notamment en 2008. Peut-être devons-nous voir dans ce résultat l'influence de la meilleure qualité des orthophotographies de 2000 et 2008 qui permettent d'identifier plus de mouvements de masse (en particulier les petits).

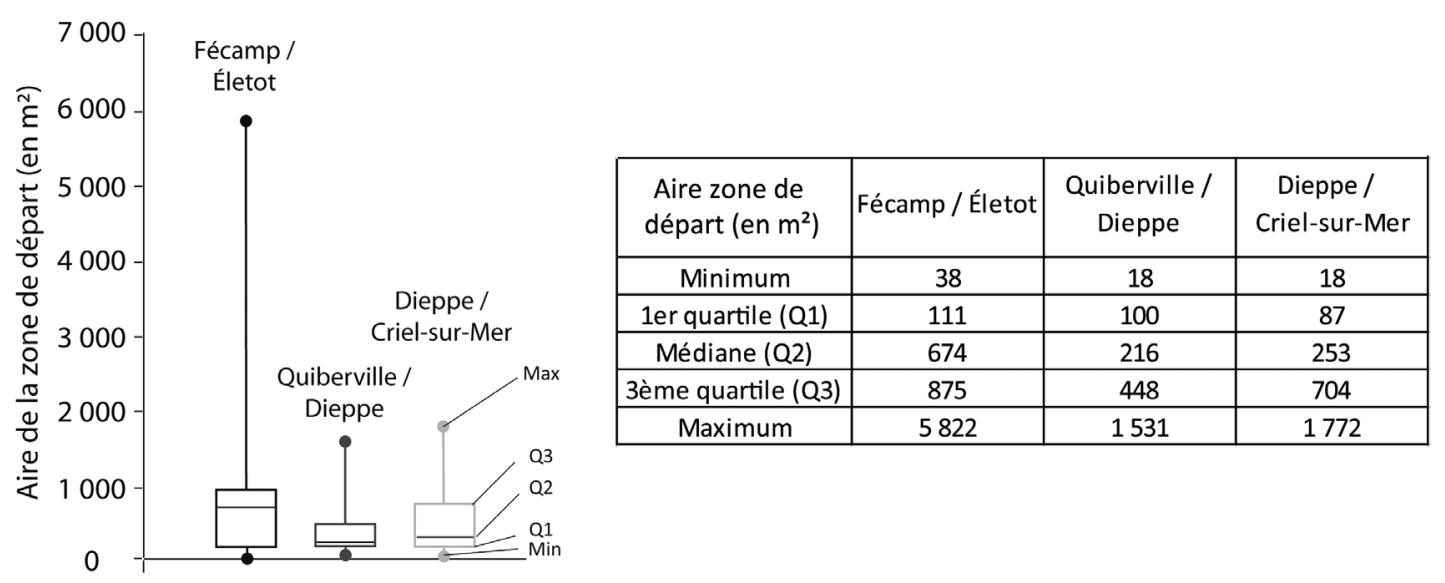

Fig. 7 - Aire des éboulements/écroulements observés entre Étretat et Le Tréport (1939- 2008).

Fig. 7 - Area of observed falls from Etretat to Le Treport (1939-2008). 
Cependant, durant l'automne 2012 et l'hiver 2013, un nombre important de mouvements de masse de grande ampleur a été observé ayant des conséquences importantes sur les populations côtières (fermeture de la rue Chewington à Criel-surMer, de la route de Pourville à Dieppe). Nous pensons que ce temps fort de la morphogénèse fait plus appel à la variabilité qu'à une tendance à long terme.

L'analyse conjointe des vitesses et des modalités du recul des falaises fait apparaître au moins deux secteurs aux dynamiques distinctes. Ainsi, comme il a été évoqué précédemment, le premier secteur se caractérise par des vitesses de recul faibles à modérées mais les éboulements/écroulements y sont massifs (aires de départ pouvant atteindre $5800 \mathrm{~m}^{2}$ à Fécamp/Életot et Dieppe/Criel-sur-Mer, avec un recul moyen par événement de 9 à $10 \mathrm{~m}$ ). Le second secteur (Saint-Valéry-en-Caux/Dieppe) est affecté par des vitesses de recul plus importantes. Cela est en lien avec une plus grande fréquence d'éboulements/écroulements de dimensions plus réduites (aire de départ maximale de $1500 \mathrm{~m}^{2}$ entre Quiberville et Dieppe, et un recul moyen de 7,5 m). Là encore, cette répartition spatiale des dynamiques est probablement à mettre en relation avec les caractéristiques morphostructurales du littoral haut-normand (Costa, 1997 ; Costa et al., 2004). Ainsi, la section côtière Saint-Valéry-enCaux/Dieppe (secteur au recul rapide mais avec des événements fréquents et peu intenses) se situe dans l'axe de la gouttière normande se prolongeant par le synclinal de Varengeville et la retombée péri-anticlinale de la boutonnière du Bray. Cela induit, d'une part, une hauteur réduite des falaises qui permet une évacuation rapide des mouvements de masse et donc la réactivation rapide de l'instabilité des abrupts, et d'autre part, l'affleurement des craies santoniennes et campaniennes que les essais géotechniques qualifient de peu résistantes (Laignel, 1997).

Le recensement de terrain, réalisé de façon hebdomadaire en collaboration avec l'association ESTRAN entre Veules et Le Tréport (2002-2009), a permis de répertorier 331 éboulements/écroulements. Cet inventaire autorise des traitements statistiques visant à déterminer l'occurrence des éboulements/écroulements. Ce type d'analyses a depuis longtemps montré les relations entre la magnitude et la fréquence des ruptures pour les versants rocheux quasi-verticaux. Différentes lois de distribution sont évoquées dans la littérature scientifique pour s'ajuster aux données de fréquence en fonction des volumes : loi de puissance, de Gumbel, exponentielle (Dussauge et al., 2002).
Les calculs des fréquences d'occurrence et des périodes de retour portent sur des éboulements/écroulements d'au moins $1000 \mathrm{~m}^{3}$. En effet, ces derniers affectent des pans entiers de falaise et ne relèvent pas d'une éboulisation localisée s'accompagnant de réajustements du profil de falaise après un éboulement écroulement majeur. La période de retour des éboulements/écroulements s'avère beaucoup plus rapide entre Sainte-Marguerite-sur-Mer et Varengevillesur-Mer avec une durée de retour d'un éboulement/écroulement d'au moins $1000 \mathrm{~m}^{3}$ par kilomètre linéaire tous les 6 mois, contre 2,5 ans entre Pourville-sur-Mer et Bracquemont, et 3 ans entre Veules-les-Roses et Le Tréport (fig. 8). Cependant, il convient de préciser que cette période de retour ne correspond pas à l'intervalle de temps entre deux éboulements/écroulements s'effectuant au même endroit, que certains auteurs comme H. Evrard et M. Sinelle (1980) appellent malencontreusement « cycle d'évolution des falaises ».

Concernant le rythme de recul, nous espérions, à partir du recensement des éboulements/écroulements visibles sur les missions aériennes de l'IGN, identifier des secteurs affectés par une évolution complète du recul des falaises (recul d'un pan entier de falaise, déblaiement, météorisation de l'abrupt, puis, au même endroit, un second recul de pan entier). Les résultats sont peu cohérents car les éboulements/écroulements observés au même endroit sur les photographies de l'IGN relèvent de la chance, notamment pour les petits volumes souvent évacués entre deux missions aériennes.

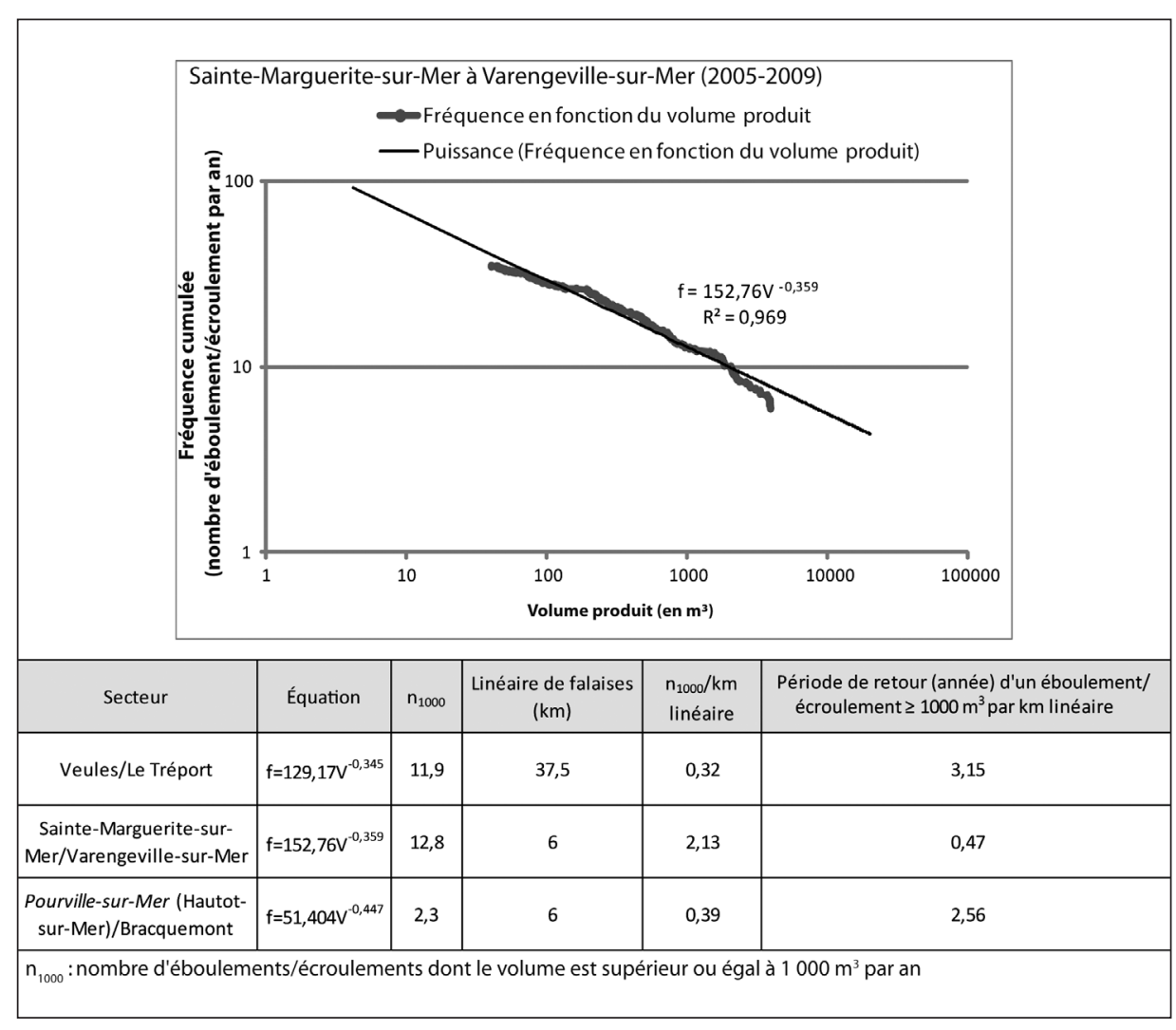

Fig. 8 - Ajustement à la loi de puissance des éboulements/écroulements recensés entre 2002 et 2009.

Fig. 8 - Fitting to power law of censed falls between 2002 and 2009. 
Cependant, pour le secteur de Mesnil-Val, T. Dewez et al. (2013) évoquent un rythme très court. En effet, un éboulement/écroulement majeur (ayant engendré un recul de $10 \mathrm{~m}$ en haut de falaise) a été précédé, quatre ans auparavant, par l'éboulement/écroulement d'un pan entier de falaise. Cette rapidité s'explique, en partie, par le fait que la section étudiée par les auteurs $(700 \mathrm{~m})$ se localise juste à l'aval-dérive de l'épi majeur de Criel-sur-Mer et de Mesnil-Val, à savoir un espace dénué de galets et donc sous l'action des agents marins ici très efficace.

Le suivi hebdomadaire de l'association ESTRAN montre également que le rythme d'évolution est assez rapide au cap d'Ailly. Ainsi, au regard des quelques cas observés entre 2002 et 2009, la période de retour se situe autour de 7 ans. Cependant, ces trois dernières années (2011 à 2013), les cas d'éboulements/écroulements successifs que nous avons observés ramènent l'occurrence autour de 5 ans. Enfin, nous avons observé un cas pour lequel trois éboulements/écroulements de pan entier de falaise se sont opérés, au même endroit, et ce, à un an d'intervalle. Le cap d'Ailly est donc un secteur où l'évacuation des éboulements/écroulements est rapide, tout comme la météorisation de la paroi.

\section{Conclusion}

Il est communément admis que les falaises crayeuses de Haute-Normandie sont affectées par un recul rapide qui se fait par à-coups et sur des pas de temps longs. Les méthodes utilisées dans ce travail ont donc pour objectif d'apporter des éléments quantitatifs, notamment sur les vitesses, les modalités, voire les rythmes de recul.

Parmi les principaux résultats, notamment méthodologiques, il s'avère que pour les côtes rocheuses, la méthode de mesure du retrait fondée sur l'aire (surface perdue en haut de l'abrupt crayeux) semble la plus adaptée. Ainsi, entre 1966 et 2008, le littoral haut-normand a connu un recul moyen de $0,15 \mathrm{~m} / \mathrm{an}( \pm 0,03 \mathrm{~m} / \mathrm{an})$. Cette valeur moyenne est peu représentative puisqu'il existe une très grande variabilité spatiale des vitesses de retrait, notamment à grande échelle (tous les $50 \mathrm{~m}$ ) allant de quelques $\mathrm{cm}$ à $80 \mathrm{~cm} / \mathrm{an}$. La répartition spatiale de ces vitesses semble correspondre aux caractéristiques lithologiques régionales. Le recul est faible à modéré (de 0,09 à 0,18 m/an) entre Antifer/Paluel et Dieppe/Le Tréport, où affleurent les craies plus résistantes de la base du Coniacien, du Turonien, voire du Cénomanien. Le second secteur est caractérisé par un recul plus important $(0,23 \mathrm{~m} / \mathrm{an})$, entre Saint-Valéry-en-Caux et Dieppe, où affleurent le Santonien et le Campanien. Cette répartition spatiale des vitesses de recul peut localement être perturbée par des obstacles au transit des galets. Ainsi, à l'aval-dérive immédiat de ces derniers (jetées portuaires, notamment), les vitesses moyennes peuvent être multipliées par 3 ou 4. L'étude conjointe des vitesses de retrait et des caractéristiques morphologiques des éboulements/écroulements (zone de départ) à partir des photographies aériennes de l'IGN apporte une plus-value à ces conclusions. Les secteurs affectés par un recul important sont caractérisés par des éboulements/écroulements fréquents et peu volumi- neux, alors que ceux affectés par un recul faible sont caractérisés par des éboulements/écroulements volumineux et rares. S'agissant des rythmes d'évolution des falaises, ils sont difficilement identifiables à partir des photographies aériennes verticales de l'IGN. En revanche, le suivi de terrain montre que dans les secteurs à recul rapide, les rythmes (entre deux mouvements de pan entiers de falaise s'effectuant au même endroit) sont de l'ordre de 5 à 7 ans au cap d'Ailly. Ces résultats peuvent être nettement améliorés dès qu'il sera possible d'augmenter l'intervalle de temps et la fréquence d'analyse, et ce, avec des données haute résolution (levés LIDAR aéroporté, terrestre ou embarqué). Cette précision permettra de prendre en compte les petits éboulements/écroulements, évacués en quelques marées, qui représentent près de $11 \%$ du recul total sur les côtes crayeuses du Sussex (May et Heeps, 1985). Par ailleurs, à propos des facteurs explicatifs de la répartition spatiale des vitesses de recul, au-delà de la lithodépendance, il conviendra désormais de prendre en considération les facteurs internes prédisposants tels que la fracturation de la craie. Ce facteur, non considéré dans ce travail, détermine probablement la résistance des divers étages de craie et les caractéristiques morphologiques (et donc le volume) des éboulements / écroulements.

\section{Références}

Bialek J. (1969) - Le recul des falaises du cap d'Antifer au Tréport, entre 1830 et 1966. Direction Départementale de l'Équipement de Seine-Maritime, Arrondissement de Dieppe, 63 p.

Bignot G. (1962) - Étude sédimentologique et micropaléontologique de l'Éocène du Cap d'Ailly (près de Dieppe-Seine-Maritime). Thèse, Université de Paris, $260 \mathrm{p}$.

Costa S. (1997) - Dynamique littorale et risques naturels : L'impact des aménagements, des variations du niveau marin et des modifications climatiques entre la Baie de Seine et la Baie de Somme. Thèse, Université de Paris I, 376 p.

Costa S. (2000) - Réactualisation des connaissances et mise en place d'une méthode de suivi de la dynamique du littoral hautnormand et picard. Rapport final, Préfecture de Picardie, Contrat de Plan Interrégional du Bassin de Paris (CPIBP), 103 p.

Costa S., Delahaye D., Freiré-Diaz S., Di Nocera L., Davidson, R., Plessis, E. (2004) - Quantification of the Normandy and Picardy chalk cliff retreat by photogrammetric analysis. In Mortimore R.N., Duperret A. (Eds.) : Coastal Chalk Cliff Instability. Engineering Geology Special Publications, 20, 139-148.

Costa S., Laignel B., Hauchard E., Delahaye D. (2006) - Facteurs de répartition des entonnoirs de dissolution dans les craies du littoral du Nord-Ouest du Bassin de Paris. Zeitschrift für Geomorphologie 50, 1, 95-116.

Dewez T.J.B., Rohmer J., Regard V., Cnudde C. (2013) - Probabilistic coastal cliff collapse hazard from repeated terrestrial laser surveys: case study from Mesnil Val (Normandy, northern France). In Conley D.C., Masselink G., Russell P.E., O'Hare T.J. (Eds.): Proceedings of the 12th International Coastal Symposium (Plymouth, England). Journal of Coastal Research, Special Issue 65, 702-707. 
Dolan R., Hayden B., Hejrwood J. (1978) - A new photogrammetric method for determining shoreline erosion. Coastal Engineering 2, 21-39.

Dornbusch U., Robinson D.A., Moses C.A., Williams R.B.G. (2008a) - Temporal and spatial variations of the chalk cliff retreat rate in East Sussex, 1873-2001. Marine Geology 249, 271-282.

Dornbusch U., Robinson D.A., Moses C.A., Williams R.B.G. (2008b) - Soft copy photogrammetry to measure shore platform erosion on decadal timescales. Journal of Coastal Conservation $11,193-200$

Dussauge-Peisser C., Helmstetter A., Grasso J.R., Hantz D., Desvarreux P., Jeannin M., Giraud A. (2002) - Probabilistic approach to rock fall hazard assessment: potential of historical data analysis. Natural Hazard and Earth System Sciences 2, $1-13$.

Évrard H., Sinelle M. (1980) - Stabilité des falaises du Pays de Caux. Rapport CETE, Seine-Maritime, 85 p.

Faye I.B.N., Giraudet E., Gourmelon F., Hénaff A. (2012) Cartographie normalisée de l'évolution du trait de côte. $M @ p p e-$ monde, 104(2011.4), $12 \mathrm{p}$.

Foyle A.M., Naber M.D. (2012) - Decade-scale coastal bluff retreat from LiDAR data: Lake Erie coast of NW Pennsylvania, USA. Environmental Earth Sciences 66, 1999-2012.

Hénaff A., Lageat Y., Costa S., Plessis E. (2002) - Le recul des falaises crayeuses du Pays de Caux : détermination des processus d'érosion et quantification des rythmes d'évolution. Géomorphologie : Relief, Processus, Environnement, 2, 107-118.

IGN (2008) - BD Ortho®.

Juignet P., Breton G. (1992) - Mid-Cretaceous sequence stratigraphy and sedimentary cyclicity in the western Paris Basin. Palaeogeography, Palaeoclimatology, Palaeoecology 91, 3, 197-218.

Lahousse P., Pierre G. (2003a) - The retreat of chalk cliffs at Cap Blanc-Nez (France): autopsy of an erosional crisis. Journal of Coastal Research 19, 2, 431-440.

Lahousse P., Pierre G. (2003b) - Vitesse, rythme et modalités du recul des falaises crayeuses du cap Blanc-Nez. Zeitschrift für Geomorphologie 47, 2, 215-234

Laignel B. (1997) - Les altérites à silex de l'ouest du Bassin de Paris : caractérisation lithologique, genèse et utilisation potentielle comme granulats. Thèse, Université de Rouen, 219 p.

Lamblardie (De) J.E. (1789) - Mémoire sur les côtes de la HauteNormandie comprise entre l'embouchure de la Seine et celle de la Somme, considérées relativement au galet qui remplit les ports situés dans cette partie de la Manche. Bulletin de la Société Géologique de Normandie, 1908, 55-93.

Lautridou J.P. (1985) - Le cycle périglaciaire pléistocène en Europe du Nord-Ouest et plus particulièrement en Normandie. Thèse, Université de Caen, 908 p.

Letortu P. (2013) - Le recul des falaises crayeuses haut-normandes et les inondations par la mer en Manche centrale et orientale : de la quantification de l'aléa à la caractérisation des risques induits. Thèse, Université de Caen, 408 p.

Lim N., Petley D.N., Rosser N.J., Allison R.J., Long A.J., Pybus D. (2005) - Combined digital photogrammetry and time-offlight laser scanning for monitoring cliff evolution. The Photogrammetric Record 20, 110, 109-129.

Lim M., Rosser N.J., Allison R.J., Petley D.N. (2010) - Erosional processes in the hard rock coastal cliffs at Staithes, North Yorkshire. Geomorphology 114, 12-21.

May V., Heeps C. (1985) - The nature and rates of changes on chalk coastlines. Zeitschrift für Geomorphologie, Supplementband 57, 81-94.

Mégnien C., Mégnien F. (1980) - Synthèse géologique du Bassin de Paris. Mémoire du Bureau des Recherches Géologiques et Minière, 3 volumes, $\mathrm{n}^{\circ}$ 101, 102, 103.

Moore L.J. (2000) - Shoreline Mapping Techniques. Journal of Coastal Research 16, 1, 111-124.

Overton M., Petrina C., Fisher J. (1996) - Determining shoreline position using historical photography and digital soft-copy photogrammetry. ASPRS/ACSM Annual Convention And Expo. Technical Paper, 1, 512-513.

Pierre G., Lahousse P. (2006) - The role of groundwater in cliff instability: an example at Cape Blanc-Nez (Pas-de-Calais, France). Earth Surface Process and Landforms 31, 31-45.

Pomerol B., Bailey H.W., Monciardini C., Mortimore R.N. (1987) - Lithostratigraphy and biostratigraphy of the Lewes and Seaford chalks: a link across the Anglo-Paris basin at the Turonian-Senonian boundary. Cretaceous Research 8, 289-304.

Regard V., Dewez T., Bourles D.L., Anderson R. S., Duperret A., Costa S., Leanni L., Lasseur E., Pedoja K., Maillet G.M. (2012) - 6 kyrs of sea-cliff retreat recorded by ${ }^{10} \mathrm{Be}$ : Theory and example from the English Channel. Quaternary Geochronology $11,87-97$.

Robin M. (2002) - Télédétection et modélisation du trait de côte et de sa cinématique.In Baron-Yelles N., Goeldner-Gianella L., Velut S. (Eds.) : Le Littoral, regards, pratiques et savoirs. Presses universitaire de l'École Normale Supérieure, 95-115.

Rodet J. (1991) - Les karsts de la craie: étude comparative. Thèse d'État, Université de Paris, 562 p.

Thieler E.R., Himmelstoss E.A., Zichichi J.L., Miller T.L. (2005) - Digital Shoreline Analysis System (DSAS) version 3.0: An ArcGIS extension for calculating shoreline change. US Geological Survey Open-File Report 2005-1304.

Young A. P., Flick R.E., Gutierrez R., Guza R.T. (2009) - Comparison of short-term seacliff retreat measurement methods in Del Mar, California. Geomorphology 112, 318-323.

Zviely D., Klein M. (2004) - Coastal cliff retreat rates at Beit-Yannay, Israel, in the 20th century. Earth Surface Processes and Landforms 29, 175-184.

Article soumis le 24 mai 2013, accepté le 23 février 2014. 